\title{
Nonlinear Modeling and Control Design of Active Helicopter Blades
}

\author{
Matthias Althoff;, Mayuresh J. Patil ${ }^{\dagger}$ \\ Virginia Polytechnic Institute and State University, Blacksburg, VA 24061-0203 \\ and \\ Johannes P. Traugott $\ddagger$ \\ Technische Universität München, D-85747 Garching, Germany
}

\begin{abstract}
The paper presents the theoretical basis for the simulation of active helicopter blades. The analysis of active helicopter blades is based on models for the structure, aerodynamics, actuation and sensing. For simulation and analysis purposes, the blade structural model is discretized in space using a Galerkin approach. The effect of the integral actuation enters the beam model via an active beam cross-sectional analysis. A 2-D incompressible, inviscid, quasi-steady aerodynamic model is coupled to the active structural model. The resulting nonlinear model of high order is reduced using the aeroelastic modes of the blade. Finally, a nonlinear controller is obtained by cancelling the nonlinearities and applying an energy optimal LQR design to the resulting linear plant.
\end{abstract}

\section{Introduction}

Active structures have the potential to outperform conventional structures in many ways. In the case of helicopter blades, active structures can overcome the compromise between vibration and weight reduction. ${ }^{1,2}$ This potential has been investigated in tests of the Active Twist Rotor (ATR) blade. The paper presents theoretical results of a rotor blade based on the parameters of the ATR. The present paper is a continuation of the research presented in Traugott et al. ${ }^{3}$ dealing with the nonlinear dynamic solution and control design of active helicopter blades. Following are the significant new developments:

- A structural dynamic model based on a nonlinear Galerkin approach which is more efficient ${ }^{4}$ as compared to FEM is used

- A quasi-steady aerodynamic model is included

- A nonlinear model order reduction technique is used to derive a low-order, high fidelity nonlinear blade model for control design

- Nonlinear control design is investigated

The paper is organized in seven sections. In section II the blade model is introduced followed by the Galerkin approach for spatial discretization in section III. Modal analysis is presented in section IV and the normal modes are utilized for model order reduction in section V. The control design is discussed in section VI and the paper ends with conclusions in section VII.

\section{Blade Model}

Accurate modeling of active helicopter blade dynamics requires the merger of the physical models of different domains which affect the dynamics of an active helicopter blade. The four models which need

${ }^{*}$ Visiting Student, Department of Aerospace and Ocean Engineering. Presently, Dipl.-Ing., Institute of Automatic Control, Technische Universität München, Germany.

${ }^{\dagger}$ Assistant Professor, Department of Aerospace and Ocean Engineering. Senior Member AIAA.

${ }^{\ddagger}$ Dipl.-Ing., Institute of Flight Mechanics and Flight Control. 
to be developed and integrated are: the structural dynamic model, the aerodynamic model, the actuation model and the sensing model of the helicopter blade. The structural and aerodynamic models are inherently nonlinear. The presented models are an extension of earlier work done by the authors. ${ }^{4,5}$

\section{A. Structural Model}

In order to calculate the dynamics of the helicopter blade at low computational costs, a nonlinear beam model developed by Hodges ${ }^{6,7}$ is used. This model takes advantage of the one dimensional characteristics of a helicopter blade and is a better choice compared to 3-D finite-element analysis. ${ }^{8}$ It models a beam undergoing large deformation and small strain. The beam formulation is intrinsic, i.e. neither displacement nor rotation variables appear in the beam equations. The intrinsic formulation is very compact and furthermore applicable for general beams (anisotropic, non-uniform, twisted and curved).

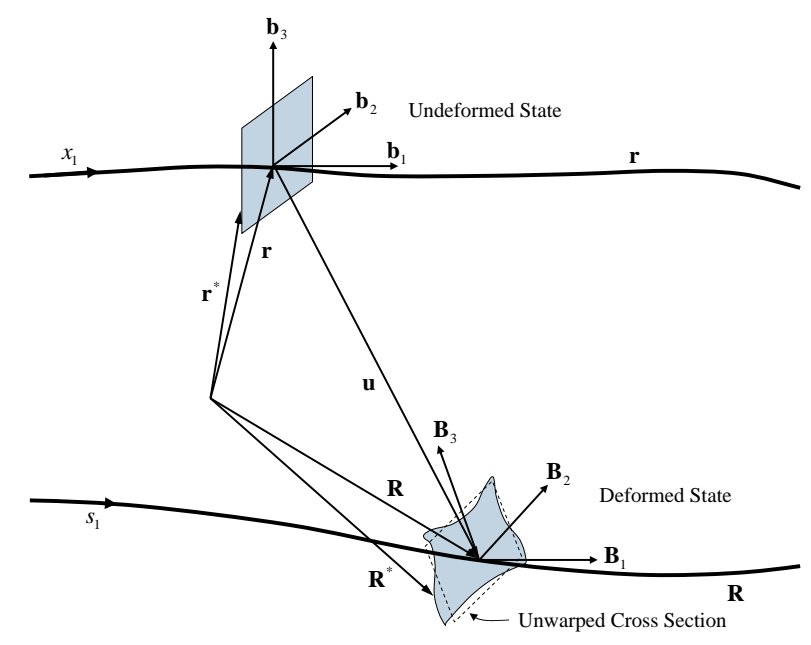

Figure 1. Schematic of beam undergoing finite deformation and cross-sectional warping

The measure numbers of the variables in the beam model are calculated in the deformed frame (B-frame). This B-frame is orthogonal and defined by the cross section of the deformed beam as seen in Figure 1 . The $B_{2}$-axis and the $B_{3}$-axis lie in the cross-section with the $B_{1}$-axis defined by $B_{1}=B_{2} \times B_{3}$.

The intrinsic equations for the nonlinear dynamics of the beam are:

$$
\begin{aligned}
F^{\prime}+(\tilde{\mathbb{k}}+\tilde{\kappa}) F+f^{\text {aero }}+f^{\text {dist }} & =\dot{P}+\tilde{\Omega} P \\
M^{\prime}+(\tilde{\mathbb{k}}+\tilde{\kappa}) M+\left(\tilde{e}_{1}+\tilde{\gamma}\right) F+m^{\text {aero }}+m^{\text {dist }} & =\dot{H}+\tilde{\Omega} H+\tilde{V} P \\
V^{\prime}+(\tilde{\mathbb{k}}+\tilde{\kappa}) V+\left(\tilde{e}_{1}+\tilde{\gamma}\right) \Omega & =\dot{\gamma} \\
\Omega^{\prime}+(\tilde{\mathbb{k}}+\tilde{\kappa}) \Omega & =\dot{\kappa}
\end{aligned}
$$

where, ()$^{\prime}$ denotes the derivative with respect to the beam reference line and $(\dot{)})$ denotes the absolute time derivative. $F$ and $M$ are the measure numbers of the internal force and moment vector (generalized forces), $P$ and $H$ are the measure numbers of the linear and angular momentum vector (generalized momenta), $\gamma$ and $\kappa$ are the beam strains and curvatures (generalized strains), $V$ and $\Omega$ are the linear and angular velocity measures (generalized velocities). The external forces and moments due to aerodynamic effects are $f^{a e r o}$, $m^{\text {aero }}$ and due to disturbances are $f^{\text {dist }}, m^{\text {dist }}$ respectively. $k_{k}=\left\lfloor\begin{array}{llll}k_{1} & k_{2} & k_{3}\end{array}\right\rfloor$ is the initial twist/curvature of the beam and $e_{1}=\left\lfloor\begin{array}{lll}1 & 0 & 0\end{array}\right\rfloor^{T}$. The tilde operator transforms a vector $a$ to a matrix $\widetilde{a}$ so as to effect a cross product when left-multiplied to the vector $b$, i.e., $\widetilde{a} b=a \times b$.

The intrinsic beam equations provide four vector equations for eight vector unknowns $(F, M, P, H, \gamma, \kappa$, $V, \Omega)$. In order to complete a solvable set of equations, four more vector equations are needed. Two equations relate the generalized forces $(F, M)$ and the generalized strains $(\gamma, \kappa)$ via the beam cross-section stiffness matrix. The beam cross-section inertia matrix leads to the relation between the generalized momenta $(P$, 
$H)$ and the generalized velocities $(V, \Omega)$. Both relations are the constitutive equations for an active beam and derived from an accurate cross-sectional analysis:

$$
\begin{aligned}
& \left\{\begin{array}{l}
F \\
M
\end{array}\right\}=\left[\begin{array}{ll}
\mathbb{R} & \mathbb{S} \\
\mathbb{S}^{T} & \mathbb{T}
\end{array}\right]^{-1}\left\{\begin{array}{l}
\gamma \\
\kappa
\end{array}\right\}-\left\{\begin{array}{l}
F^{A} \\
M^{A}
\end{array}\right\} \\
& \left\{\begin{array}{l}
P \\
H
\end{array}\right\}=\left[\begin{array}{cc}
\mathbb{G} & \mathbb{K} \\
\mathbb{K}^{T} & \mathbb{\mathbb { V }}
\end{array}\right]\left\{\begin{array}{l}
V \\
\Omega
\end{array}\right\}
\end{aligned}
$$

where, $\mathbb{R}, \mathbb{S}$ and $\mathbb{T}$ are the cross-sectional flexibilities, and $F^{A}, M^{A}$ are generalized forces induced by the active elements. The cross-sectional flexibilities and the induced generalized forces can be obtained by conducting active cross-sectional analysis using the theory of Patil and Johnson ${ }^{9}$ (for thin-walled beams) or Cesnik and Palacios ${ }^{10}$ (for general configuration). The inertia matrices have the following components:

$$
\mathbb{G}=\mu I=\left[\begin{array}{ccc}
\mu & 0 & 0 \\
0 & \mu & 0 \\
0 & 0 & \mu
\end{array}\right], \quad \mathbb{K}=-\mu \tilde{\xi}=\left[\begin{array}{ccc}
0 & \mu \tilde{\xi}_{3} & -\mu \tilde{\xi}_{2} \\
-\mu \tilde{\xi}_{3} & 0 & 0 \\
\mu \tilde{\xi}_{2} & 0 & 0
\end{array}\right], \quad \mathbb{\square}=\left[\begin{array}{ccc}
\AA_{2}+\AA_{3} & 0 & 0 \\
0 & \AA_{2} & \AA_{23} \\
0 & \AA_{23} & \AA_{3}
\end{array}\right]
$$

where, $\mu, \xi, \stackrel{\circ}{0}_{2}, \stackrel{\circ}{3}_{3}, \stackrel{\circ}{2}_{23}$ are the mass per unit length, mass center offset and the three cross-sectional mass momenta of inertia per unit length.

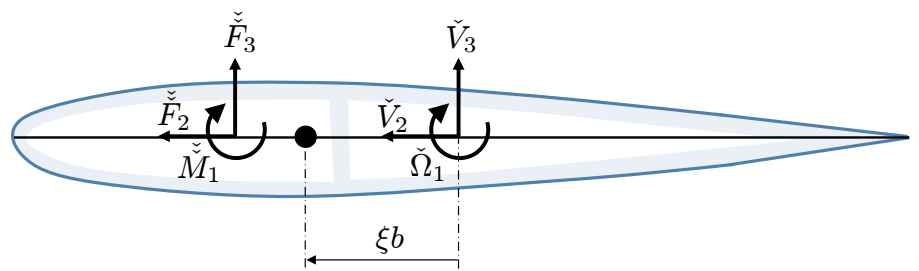

Figure 2. Velocities and aerodynamic forces in the blade profile

\section{B. Aerodynamic Model}

To take into account the primary aeroelastic effects, an incompressible, inviscid, quasi-steady, 2-D aerodynamic model ${ }^{5}$ based on a finite-state airload model ${ }^{11}$ is used. The model neglects the unsteady effects due to the wake. The aerodynamic loads $f^{\text {aero }}$ and $m^{\text {aero }}$ in Eq. (1) are calculated using:

$$
\begin{aligned}
& f^{\text {aero }}=\left\{\begin{array}{c}
0 \\
-\rho b C_{l 0} \check{V}_{2} \check{V}_{3}+\rho b C_{l \alpha} \check{V}_{3}^{2}-\rho b C_{d 0} \check{V}_{2}^{2} \\
\rho b C_{l 0} \check{V}_{2}^{2}-\rho b\left(C_{l \alpha}+C_{d 0}\right) \check{V}_{2} \check{V}_{3}+\frac{1}{2} \rho b^{2} C_{l \alpha} \check{V}_{2} \Omega_{1}
\end{array}\right\} \\
& \check{m}^{\text {aero }}=\left\{\begin{array}{c}
2 \rho b^{2} C_{m 0} \check{V}_{2}^{2}-\frac{1}{4} \rho b^{3} C_{l \alpha} \check{V}_{2} \Omega_{1} \\
0 \\
0
\end{array}\right\}
\end{aligned}
$$

where, $(\check{)}$ ) denotes a variable measured at mid-chord and $(\check{)})$ denotes a variable calculated at quarter-chord. $\rho$ is the air density and $b$ is the semi-chord. Moreover, one can see from Figure 2 that:

$$
\begin{aligned}
\check{V}_{3} & =V_{3}-\xi b \Omega_{1}, \quad \check{V}_{2}=V_{2} \\
m_{1}^{\text {aero }} & =\check{m}_{1}^{\text {aero }}+(0.5-\xi) b f_{3}^{\text {aero }}
\end{aligned}
$$

After inserting Eq. (5) into Eq. (4), the new equations can be written in terms of $V$ and $\Omega$ as:

$$
\begin{aligned}
f^{\text {aero }} & =\left\{\begin{array}{lll}
0 & 1 & 0
\end{array}\right\}^{T}\left[V^{T} \mathbb{K}^{V V} V+V^{T} \mathbb{K}^{V \Omega} \Omega+\Omega^{T} \mathfrak{X}^{\Omega \Omega} \Omega\right]+\left\{\begin{array}{lll}
0 & 0 & 1
\end{array}\right\}^{T}\left[V^{T} \mathbb{Y}^{V V} V+V^{T} \mathbb{Y}^{V \Omega} \Omega\right] \\
m^{\text {aero }} & =\left\{\begin{array}{lll}
1 & 0 & 0
\end{array}\right\}^{T}\left[V^{T} \mathbb{Z}^{V V} V+V^{T} \mathbb{Z}^{V \Omega} \Omega\right]
\end{aligned}
$$


where, the matrices used above are defined as:

$$
\begin{aligned}
& \mathbb{Y}^{V V}=\rho b\left[\begin{array}{ccc}
0 & 0 & 0 \\
0 & C_{l 0} & -\left(C_{l \alpha}+C_{d 0}\right) \\
0 & 0 & 0
\end{array}\right], \quad \quad \mathbb{X}^{V V}=\quad \rho b\left[\begin{array}{ccc}
0 & 0 & 0 \\
0 & -C_{d 0} & -C_{l 0} \\
0 & 0 & C_{l \alpha}
\end{array}\right] \\
& \mathbb{Y}^{V \Omega}=\rho b^{2}\left[\begin{array}{ccc}
0 & 0 & 0 \\
(0.5+\xi) C_{l \alpha}+\xi C_{d 0} & 0 & 0 \\
0 & 0 & 0
\end{array}\right], \quad \quad \mathbb{R}^{V \Omega}=\quad \xi \rho b^{2}\left[\begin{array}{ccc}
0 & 0 & 0 \\
C_{l 0} & 0 & 0 \\
-2 C_{l \alpha} & 0 & 0
\end{array}\right] \\
& \mathbb{Z}^{V V}=\quad \rho b^{2}\left[\begin{array}{ccc}
0 & 0 & 0 \\
0 & 2 C_{m 0}+(0.5-\xi) C_{l 0} & -(0.5-\xi)\left(C_{l \alpha}+C_{d 0}\right) \\
0 & 0 & 0
\end{array}\right], \quad \chi^{\Omega \Omega}=\xi^{2} \rho b^{3}\left[\begin{array}{ccc}
C_{l \alpha} & 0 & 0 \\
0 & 0 & 0 \\
0 & 0 & 0
\end{array}\right] \\
& \mathbb{Z}^{V \Omega}=\xi \rho b^{3}\left[\begin{array}{ccc}
0 & 0 & 0 \\
-\xi C_{l \alpha}+(0.5-\xi) C_{d 0} & 0 & 0 \\
0 & 0 & 0
\end{array}\right]
\end{aligned}
$$

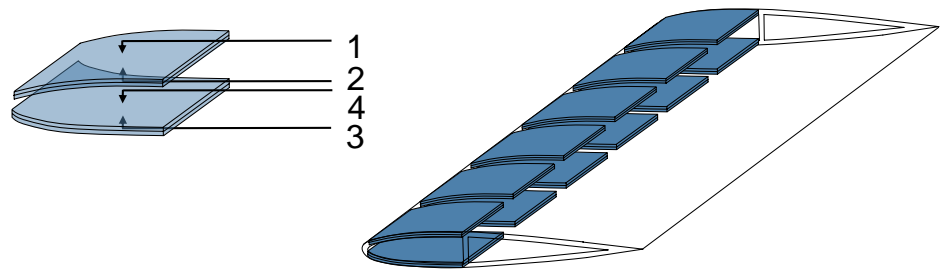

Figure 3. Actuator distribution in an integrally actuated blade

\section{Actuation Model}

The actuation in an active helicopter blade like the Active Twist Rotor (ATR) is provided by active fiber composites (AFC) that are distributed as discrete segments over the span of the blade (see Figure 3). Each segment may contain multiple layers of AFCs which can be controlled independently (ATR contains four) and induce constant generalized forces $F^{A}$ and $M^{A}$ in the blade structure (see Eq. (2)). The generalized active forces are linearly related to the applied voltages as:

$$
\left\{\begin{array}{c}
F_{u}^{A} \\
M_{u}^{A}
\end{array}\right\}=\left[\begin{array}{cc}
\mathbb{R} & \mathbb{S} \\
\mathbb{S}^{T} & \mathbb{T}
\end{array}\right]^{-1}\left[\begin{array}{c}
\mathbb{E} \\
\mathbb{E}
\end{array}\right] u_{u}
$$

where, $u_{u}$ is the applied voltage vector for one blade segment consisting of multiple voltages. $\mathbb{E}$ and $\mathbb{E}$ are constants relating the applied voltage to the active generalized strains which are transformed to the active generalized forces via the cross-section stiffness matrix.

\section{Sensing Model}

The helicopter blade is assumed to be equipped with a number of equidistantly distributed sensors which measure the generalized strains $\gamma$ and $\kappa$ along the blade reference line. The results presented in the later sections assume five sensor locations. Furthermore, the sensors are assumed to relate the generalized strains linearly to the voltage outputs as:

$$
y_{y}=\left[\begin{array}{ll}
\mathbb{0} & \mathbb{P}
\end{array}\right]\left\{\begin{array}{l}
\gamma\left(x=\frac{y-1}{4} \mathbb{L}\right) \\
\kappa\left(x=\frac{y-1}{4} \mathbb{L}\right)
\end{array}\right\}
$$

where, $y_{y}$ denotes the measured voltages. $\mathbb{O}$ and $\mathbb{P}$ relate the sensed voltages to the generalized strains $\gamma$ and $\kappa$. Without loss of generality, the sensor matrices are simplified to $\mathbb{O}=I$ and $\mathbb{P}=I$, where, $I \in \mathbb{R}^{3 \times 3}$ is the identity matrix. 


\section{Galerkin Discretization}

The helicopter blade model equations (Eqs. (1), (2), (6), (8), (9)) form a solvable set with $V, \Omega, \kappa$ and $\gamma$ as unknowns. For simulation and analysis purposes, this equation set is discretized with respect to space. Unlike the most common approach based on the finite element method, the helicopter blade model is discretized using a Galerkin approach. ${ }^{12}$ By using special weighting functions in the Galerkin approach presented here, the approximated solution fulfills the law of energy conservation if no active elements are modeled. ${ }^{4}$ If active elements are used the law of energy conservation can be fulfilled approximately. Additionally, the boundary conditions of the problem are satisfied weakly in the Galerkin approach.

\section{A. Brief Introduction to Galerkin Discretization}

For readers who are not familiar with the Galerkin approach, a brief introduction is presented in this subsection. For a given partial differential equation the variable $w(x, t)$, which is dependent on space and time, is approximated by separation of variables in time and space as a time varying linear combination of multiple spatial function (assumed modes).

$$
f\left(w(x, t), \dot{w}(x, t), w^{\prime}(x, t), x, t\right)=0 \stackrel{w(x, t)=\sum_{i=1}^{n} \Phi_{i}(x) q_{i}(t)}{\longrightarrow} f\left(\Phi_{i}(x), \Phi_{i}^{\prime}(x), q_{i}(t), \dot{q}_{i}(t), x, t\right) \approx 0
$$

The right relation in Eq. (10) is not equal to zero in general. Now, it is required that for each $k$, the integral of Eq. (10) weighted by $\Psi_{k}(x)$ has to be zero.

$$
\int_{0}^{L}\left\{\Psi_{k}(x) f\left(\Phi_{i}(x), \Phi_{i}^{\prime}(x), q_{i}(t), \dot{q}_{i}(t), x, t\right)\right\} d x \stackrel{!}{=} 0 ;
$$

The final equations (Eq. (11)) are only ordinary differential equations in time as the integral eliminated the space dependent part. If $k, i \rightarrow \infty$, the Galerkin approximation solves the original partial differential equation exactly. The $\Phi_{i}$ are called assumed modes, the $\Psi_{k}$ are weighting functions and the $q_{i}$ are time functions.

\begin{tabular}{rll}
\hline \hline Change of kinetic energy $\quad \dot{T}$ & $=\int_{0}^{L}\left\{V^{T} \dot{P}+\Omega^{T} \dot{H}\right\} d x$ \\
Change of potential energy $\quad \dot{U}$ & $=\int_{0}^{L}\left\{\left(F+F^{A}\right)^{T} \dot{\gamma}+\left(M+M^{A}\right)^{T} \dot{\kappa}\right\} d x$ \\
External power & $P^{e x t}$ & $=\int_{0}^{L}\left[V^{T} f+\Omega^{T} m\right] d x$ \\
Boundary power & $P^{\text {bou }}$ & $=V(\mathbb{L})^{T} F^{\mathbb{L}}+\Omega(\mathbb{L})^{T} M^{\mathbb{L}}-F(\mathbb{O})^{T} V^{\mathbb{Q}}-M(\mathbb{O})^{T} \Omega^{\mathbb{Q}}$ \\
Estimated actuation power $\quad P^{a c t^{*}}$ & $\approx \int_{0}^{L}\left\{F^{A^{T}} \dot{\gamma}+M^{A^{T}} \dot{\kappa}\right\} d x$ \\
\hline \hline
\end{tabular}

Table 1. Energies in an active helicopter blade

\section{B. Energy Optimal Weighting}

In this subsection, the physical interpretation for a special choice of weighting functions for the Galerkin discretization of the blade model is given. Without loss of generality, the beam is assumed to be cantilevered and thus the boundary conditions of the blade are chosen to constrain the generalized velocities at the root $(x=\mathbb{0})$ and the generalized forces at the tip $(x=\mathbb{L})$ of the beam. Consider the following weighted sum of 
all the differential equations from Eq. (1) and its boundary conditions:

$$
\begin{aligned}
& 0=\int_{\mathbb{0}}^{\llbracket}\{ \\
& V^{T}\left[\dot{P}+\widetilde{\Omega} P-F^{\prime}-(\widetilde{\mathbb{k}}+\widetilde{\kappa}) F-f\right] \\
& +\Omega^{T}\left[\dot{H}+\widetilde{\Omega} H+\widetilde{V} P-M^{\prime}-(\widetilde{\mathbb{k}}+\widetilde{\kappa}) M-\left(\widetilde{e_{1}}+\widetilde{\gamma}\right) F-m\right] \\
& +\left(F+F^{A}\right)^{T}\left[\dot{\gamma}-V^{\prime}-(\widetilde{\mathbb{k}}+\widetilde{\kappa}) V-\left(\widetilde{e_{1}}+\widetilde{\gamma}\right) \Omega\right] \\
& +\left(M+M^{A}\right)^{T}\left[\dot{\kappa}-\Omega^{\prime}-(\widetilde{\mathbb{k}}+\widetilde{\kappa}) \Omega\right] \\
& \} d x \\
& -\left(F(\mathbb{O})+F^{A}(\mathbb{O})\right)^{T}\left[V(\mathbb{O})-V^{\mathbb{O}}\right]-\left(M(\mathbb{O})+M^{A}(\mathbb{O})\right)^{T}\left[\Omega(\mathbb{O})-\Omega^{\mathbb{D}}\right] \\
& +V(\mathbb{L})^{T}\left[F(\mathbb{L})-F^{\mathbb{L}}\right] \quad+\Omega(\mathbb{L})^{T}\left[M(\mathbb{L})-M^{\mathbb{L}}\right]
\end{aligned}
$$

where, $V^{\mathbb{Q}}$ and $\Omega^{\mathbb{Q}}$ are the exact linear and angular velocities at the root, while $F^{\mathbb{2}}$ and $M^{\mathbb{Q}}$ are the force and moment at the tip. Note, that the external forces due to aerodynamic effects and disturbances in Eq. (12) are summarized as $f$ and $m$ for simplicity reasons. After integrating by parts and simplifying Eq. (12) we have:

$$
\dot{T}+\dot{U}=P^{e x t}+P^{b o u}+P^{a c t *}
$$

where, the variables are defined in Table 1 . Equation (13) states the law of energy conservation if $P^{a c t *}=0$

\begin{tabular}{|c|c|c|c|c|c|c|c|c|c|c|}
\hline$V(x, t)$ & $=$ & $\bar{V}_{l}(x) v_{l}(t)$ & $=$ & $\Phi_{l}^{V}(x) q_{l}(t)$ & $\Phi_{l}^{V}(x)$ & $=$ & $\bar{V}_{l}$ & 0 & 0 & 0 \\
\hline$\Omega(x, t)$ & $=$ & $\bar{\Omega}_{l}(x) \omega_{l}(t)$ & $=$ & $\Phi_{l}^{\Omega}(x) q_{l}(t)$ & $\Phi_{l}^{\Omega}(x)$ & $=$ & 0 & $\bar{\Omega}_{l}$ & 0 & 0 \\
\hline$\gamma(x, t)$ & $=$ & $\bar{\gamma}_{l}(x) g_{l}(t)$ & $=$ & $\Phi_{l}^{\gamma}(x) q_{l}(t)$ & $\Phi_{l}^{\gamma}(x)$ & $=$ & 0 & 0 & $\bar{\gamma}_{l}$ & 0 \\
\hline$\kappa(x, t)$ & $=$ & $\bar{\kappa}_{l}(x) k_{l}(t)$ & $=$ & $\Phi_{l}^{\kappa}(x) q_{l}(t)$ & $\Phi_{l}^{\kappa}(x)$ & $=$ & 0 & 0 & 0 & $\bar{\kappa}_{l}$ \\
\hline$[3 \times 1]$ & & {$[3 \times 3][3 \times 1]$} & & {$[3 \times 12][12 \times 1]$} & $q_{l}(t)$ & $=$ & $v_{l}(t)$ & $\omega_{l}(t)$ & $g_{l}(t)$ & $k_{l}(t)$ \\
\hline
\end{tabular}
(passive beam). For active beams $P^{a c t}{ }^{*}$ approximates the power $P^{a c t}$ generated by the active elements as:

$P^{a c t *}=\int_{0}^{L}\{F^{A^{T}} \underbrace{\left[V^{\prime}+(\widetilde{\mathbb{k}}+\widetilde{\kappa}) V+\left(\widetilde{e_{1}}+\widetilde{\gamma}\right) \Omega\right]}_{\approx \dot{\gamma}, \text { see Eq. }(1)}+M^{A^{T}} \underbrace{\left[\Omega^{\prime}+(\widetilde{\mathbb{k}}+\widetilde{\kappa}) \Omega\right]}_{\approx \dot{\kappa}, \text { see Eq. (1) }}\} d x+F^{A^{T}} \underbrace{\left[V(\mathbb{O})-V^{\mathbb{D}}\right]}_{\approx 0}+M^{A^{T}} \underbrace{\left[\Omega(\mathbb{0})-\Omega^{\mathbb{D}}\right]}_{\approx 0}$

Thus, the weighting presented above leads to an approximate energy balance for the active beam. The error of the energy balance in Eq. (13) is caused by the active elements only. If weighting functions other than those presented in Eq. (12) are used, there will be error in the energy balance caused by all values of Table 1. For this reason, the weighting functions used in this work provide the best known solution in terms of energy conservation to the best knowledge of the authors.

Table 2. Assumed spatial modes and time functions of the approximated variables

\section{Assumed Modes and Weighting Functions}

The Galerkin discretization is performed according to subsection A. The unknown variables are approximated according to Table 2 using an expansion of products of known spatial functions (assumed modes) and unknown temporal functions (generalized coordinates). Note, that a mixed matrix/tensor notation is used (the dimensions are given in the last row in the left column of Table 2) and that all variables have a common time function $q(t)$. The advantage of the common time function is that the complete discretized blade model can be written in a single equation. 
The weighting functions for the Galerkin approach are basically chosen as shown in Eq. (12). Thus the set of weighing functions are the same as the set of variables. After substituting for the weighting functions in terms of the expansions we get a single equation, but since each generalized coordinates is arbitrary, this leads to multiple equations for each set of weighing functions. Thus, multiple equations are obtained by the Galerkin integral by using each set of weighting functions, e.g. the weighting function $V(x, t)$ is replaced by a number of $\Phi_{l}^{V}(x)$.

In this work, the assumed modes for each variable and each direction are chosen identically. Due to numerical performance, orthogonal shifted Legendre functions [Ref. 13, pp. 332-357] have been used.

$$
\bar{V}_{l}(\bar{x})=\bar{\Omega}_{l}(\bar{x})=\bar{\gamma}_{l}(\bar{x})=\bar{\kappa}_{l}(\bar{x})=I P_{l}(\bar{x})
$$

where, $I$ is a $3 \times 3$ identity matrix and $\bar{x}$ is the normed coordinate of the beam reference line $\left(\bar{x}=\frac{x}{\mathbb{L}}\right)$. After applying the Galerkin approach to Eq. (12) and discretizing the measurement equation, the beam model has the following form written in matrix/tensor notation:

$$
\begin{aligned}
\mathcal{A}_{k i} \dot{q}_{i}+\mathcal{B}_{k i} q_{i}+\mathcal{C}_{k i j} q_{i} q_{j}+\mathcal{D}_{k}+\mathcal{E}_{k u} u_{u}+\mathcal{F}_{k i u} q_{i} u_{u}+f_{k}+m_{k} & =0 \\
y_{y} & =\mathcal{M}_{y i} q_{i}
\end{aligned}
$$

where,

$$
\mathcal{B}_{k i}=\mathcal{B}_{k i}^{\text {no } B C}+\mathcal{B}_{k i}^{B C}, \quad \mathcal{C}_{k i j}=\mathcal{C}_{k i j}^{s t r u c}+\mathcal{C}_{k i j}^{a e r o}, \quad \mathcal{D}_{k}=\mathcal{D}_{k}^{B C}, \quad \mathcal{E}_{k u}=\mathcal{E}_{k u}^{n o B C}+\mathcal{E}_{k u}^{B C}
$$

The expressions for the tensors in Eqs. (16) and (17) are given in appendix B.

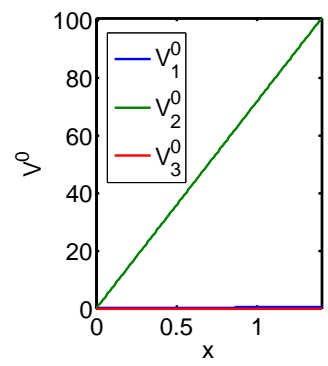

(a) Steady State of $V$

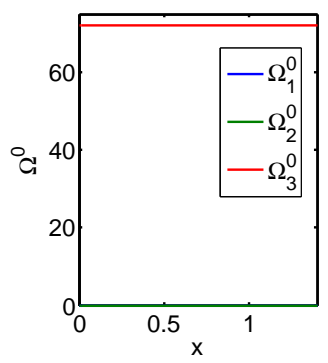

(b) Steady State of $\Omega$

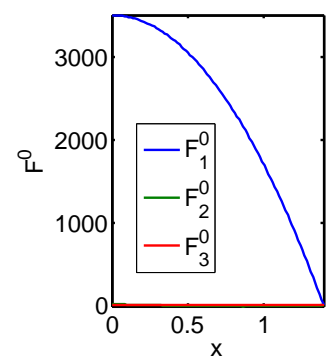

(c) Steady State of $F$

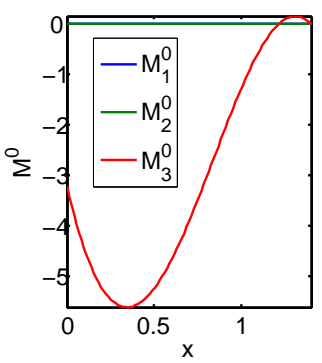

(d) Steady State of $M$

Figure 4. Steady state solution for $V, \Omega, F$ and $M$

\section{Modal Analysis}

In order to get an insight into the behavior of the helicopter blade specified in appendix A, the steady state, natural frequencies and natural modes (free vibration modes) are calculated. The steady state solution $q_{i}^{0}$ of the helicopter blade with boundary conditions specified in Eq. (12) and values $V^{\mathbb{0}}, F^{\mathbb{L}}, M^{\mathbb{L}}=0$, $\Omega^{0}=\left\{\begin{array}{lll}0 & 0 & 72\end{array}\right\}^{T} \mathrm{rad} / \mathrm{s}$ has been obtained reliably using Newton-Raphson algorithm and is presented in Figure 4 .

The natural frequencies and modes are obtained from the non-disturbed $\left(f_{k}=0, m_{k}=0\right)$, passive $\left(u_{u}=0\right)$, linearized blade model given by:

$$
\hat{\mathcal{A}}_{k i} \dot{q}_{i}+\hat{\mathcal{B}}_{k i} q_{i}=0
$$

where,

$$
\hat{\mathcal{A}}_{k i}=\mathcal{A}_{k i}, \quad \hat{\mathcal{B}}_{k i}=\mathcal{B}_{k i}+\left(\mathcal{C}_{k i j}+\mathcal{C}_{k j i}\right) q_{j}^{0}+\mathcal{F}_{k i u} u_{u}^{0}
$$

The free vibration solution (fvs) of Eq. (18) is:

$$
q_{i}^{f v s}(t)=c_{l} n_{i l} e^{\lambda_{l} t}+\hat{c}_{l} \hat{n}_{i l} e^{\hat{\lambda}_{l} t}=\mathbf{T}_{i l}\left\{e^{\lambda_{l} t} \quad e^{\hat{\lambda}_{l} t}\right\}^{T}
$$




\begin{tabular}{|c|c|c|c|c|c|}
\hline$V_{l}^{f v s}(x, t)$ & $=$ & $c_{l} V_{l}^{n m}(x)\left\{e^{\lambda_{l} t} \quad e^{\hat{\lambda}_{l} t}\right\}^{T}$ & $V_{l}^{n m}(x)$ & $=$ & $\Phi_{i}^{V}(x) \mathbf{T}_{i l}$ \\
\hline$\Omega_{l}^{f v s}(x, t)$ & $=$ & $c_{l} \Omega_{l}^{n m}(x)\left\{e^{\lambda_{l} t} \quad e^{\hat{\lambda}_{l} t}\right\}^{T}$ & $\Omega_{l}^{n m}(x)$ & $=$ & $\Phi_{i}^{\Omega}(x) \mathbf{T}_{i l}$ \\
\hline$\gamma_{l}^{f v s}(x, t)$ & $=$ & $c_{l} \gamma_{l}^{n m}(x)\left\{e^{\lambda_{l} t} \quad e^{\hat{\lambda}_{l} t}\right\}^{T}$ & $\gamma_{l}^{n m}(x)$ & $=$ & $\Phi_{i}^{\gamma}(x) \mathbf{T}_{i l}$ \\
\hline$\kappa_{l}^{f v s}(x, t)$ & $=$ & $c_{l} \kappa_{l}^{n m}(x)\left\{e^{\lambda_{l} t} \quad e^{\hat{\lambda}_{l} t}\right\}^{T}$ & $\kappa_{l}^{n m}(x)$ & $=$ & $\Phi_{i}^{\kappa}(x) \mathbf{T}_{i l}$ \\
\hline$[3 \times 1]$ & & 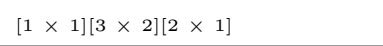 & {$[3 \times 2]$} & & {$[3 \times 12][12 \times 2]$} \\
\hline
\end{tabular}

Table 3. Free vibration solution (fvs) and natural modes (nm)

\begin{tabular}{|c|c|c|c|c|c|c|c|}
\hline \multirow{2}{*}{\multicolumn{2}{|c|}{ Mode }} & \multicolumn{2}{|c|}{ Aeroelastic Model } & \multicolumn{2}{|c|}{ NATASHA: Aeroelastic Model } & \multicolumn{2}{|c|}{ Structural Model } \\
\hline & & $\begin{array}{l}\text { frequency } \\
(\mathrm{rad} / \mathrm{s})\end{array}$ & damping & $\begin{array}{l}\text { frequency } \\
(\mathrm{rad} / \mathrm{s})\end{array}$ & damping & $\begin{array}{l}\text { frequency } \\
(\mathrm{rad} / \mathrm{s})\end{array}$ & $\begin{array}{l}\text { damping } \\
\text { damping }\end{array}$ \\
\hline $1^{s t}$ & bending & 69.4195 & $+3.26373 \cdot 10^{-1}$ & 69.4197 & $+3.26346 \cdot 10^{-1}$ & 75.9873 & $+3.08857 \cdot 10^{-13}$ \\
\hline $2^{n d}$ & bending & 196.286 & $+9.35641 \cdot 10^{-2}$ & 196.410 & $+9.34763 \cdot 10^{-2}$ & 199.654 & $-2.17091 \cdot 10^{-14}$ \\
\hline $3^{r d}$ & bending & 375.224 & $+4.30848 \cdot 10^{-2}$ & 376.198 & $+4.29394 \cdot 10^{-2}$ & 376.570 & $-1.20760 \cdot 10^{-15}$ \\
\hline $4^{t h}$ & bending & 609.286 & $+2.47827 \cdot 10^{-2}$ & 612.750 & $+2.46039 \cdot 10^{-2}$ & 610.149 & $+2.74831 \cdot 10^{-15}$ \\
\hline $5^{t h}$ & bending & 890.557 & $+1.62854 \cdot 10^{-2}$ & 899.274 & $+1.60790 \cdot 10^{-2}$ & 891.379 & $+4.23075 \cdot 10^{-15}$ \\
\hline $6^{t h}$ & bending & 1212.55 & $+1.16096 \cdot 10^{-2}$ & 1230.60 & $+1.14019 \cdot 10^{-2}$ & 1213.28 & $-7.26892 \cdot 10^{-14}$ \\
\hline $1^{s t}$ & lead-lag & 76.2633 & $+9.82787 \cdot 10^{-4}$ & 76.2685 & $+9.81159 \cdot 10^{-4}$ & 76.2633 & $+1.26594 \cdot 10^{-14}$ \\
\hline $2^{n d}$ & lead-lag & 455.697 & $+1.20758 \cdot 10^{-4}$ & 456.288 & $+1.21496 \cdot 10^{-4}$ & 455.700 & $+1.19983 \cdot 10^{-14}$ \\
\hline $3^{r d}$ & lead-lag & 1158.70 & $+4.12947 \cdot 10^{-5}$ & 1162.77 & $+4.41446 \cdot 10^{-5}$ & 1158.69 & $+1.50088 \cdot 10^{-15}$ \\
\hline $1^{s t}$ & torsion & 340.945 & $+7.47685 \cdot 10^{-2}$ & 340.972 & $+7.47336 \cdot 10^{-2}$ & 346.387 & $-6.40185 \cdot 10^{-14}$ \\
\hline $2^{\text {nd }}$ & torsion & 1019.34 & $+1.90722 \cdot 10^{-2}$ & 1020.14 & $+1.90621 \cdot 10^{-2}$ & 1021.03 & $-5.40023 \cdot 10^{-15}$ \\
\hline
\end{tabular}

Table 4. Natural frequencies of aeroelastic and structural models 


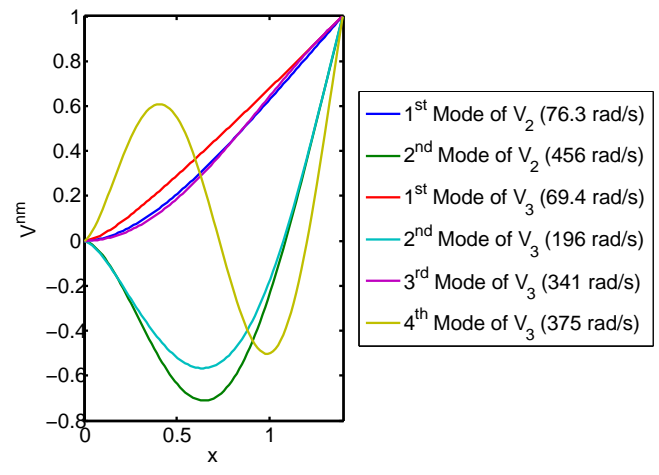

(a) Natural modes of $V$

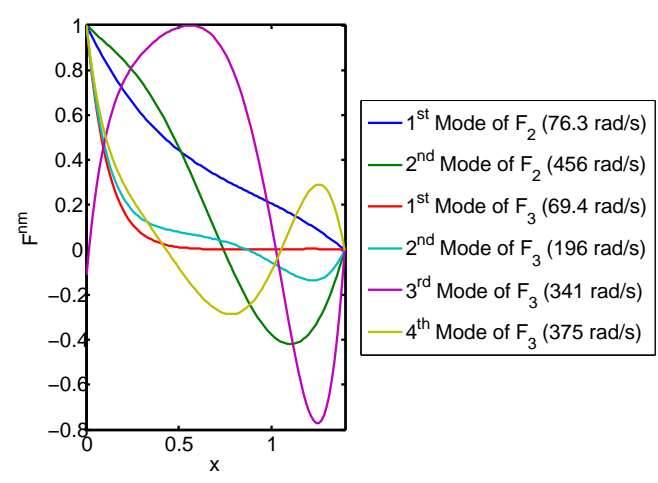

(c) Natural modes of $F$

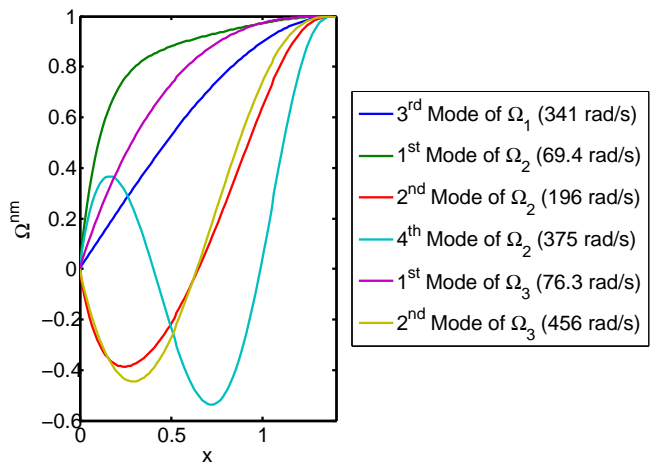

(b) Natural modes of $\Omega$

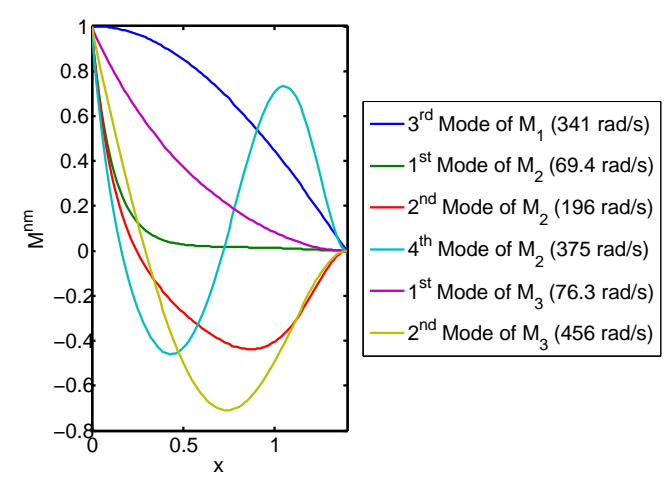

(d) Natural modes of $M$

Figure 5. Natural modes of helicopter blade

where, $\hat{()}$ denotes conjugate complex values, $c_{l}$ and $\hat{c}_{l}$ are the constant which can be calculated using the initial conditions, $n_{i l}$ and $\hat{n}_{i l}$ are the eigenvectors, $\lambda_{l}$ and $\hat{\lambda}_{l}$ denote the eigenvalues of Eq. (18), and $\mathbf{T}_{i l}=$ $\left[\begin{array}{ll}c_{l} n_{i l} & \hat{c}_{l} \hat{n}_{i l}\end{array}\right]$. Inserting Eq. (20) into Table 2 yields the natural modes of $V, \Omega, \gamma$ and $\kappa$ in Table 3 . Note, we can perform the calculations in terms of real variables by considering the real and imaginary parts of the set of complex conjugate eigenvectors. ${ }^{14}$ The natural frequencies of the helicopter blade are presented in Table 4 and are compared to results from the simulation tool NATASHA ${ }^{5}$ and to the purely structural model (no aerodynamics). For the aeroelastic and the structural model, 20 Legendre functions have been used. The NATASHA results are based on 50 beam finite elements. Firstly, the aeroelastic simulation theory presented in the paper is validated by comparing to the results generated by NATASHA. Also, one can see that the aerodynamics is mainly adding damping and reducing the frequency of the $1^{\text {st }}$ bending mode. The dominant natural modes are shown in Figure 5.

\section{Model Reduction}

The discretization in section III results in a model (Eq. (16)) of high order depending on the number of assumed modes. As both, the accuracy and the computational costs of time marching simulations increases with the number of assumed modes, one has to choose a good middle ground. A possibility to avoid a compromise is to find assumed modes that capture the dynamics of the rotating blade more accurately as compared to Legendre functions. The model order reduction follows this approach by taking low number of 
relevant assumed modes to reduce the system order without losing accuracy.

One choice of assumed modes that capture the linear dynamics are the natural modes of the system linearized at the steady state. Since these modes are used in the expansions which are applied to the complete nonlinear model, the primary nonlinear behavior is also captured. In order to improve the nonlinear prediction of the linear modes, perturbation modes ${ }^{15,16}$ can also been investigated. Although perturbation modes showed a better performance regarding the tracking of natural frequencies, they can lead to errors in the blade damping for equations in the mixed form. For this reason, only natural modes are used for order reduction. Another advantage of the natural modes is that the resulting reduced model has the same, but less natural frequencies and modes at the linearized point. Furthermore, the steady state solution is included in the new approximation of the model variables. Thus, the quality of the steady state is not affected by the order reduction and the new steady state $\mathbf{q}_{r}^{0}$ is always zero, resulting into a simple linearized reduced system.

\begin{tabular}{|c|c|c|c|c|c|c|c|c|c|}
\hline$V(x, t)$ & $=$ & $V^{0}(x)+\boldsymbol{\Phi}_{r}^{V}(x) \mathbf{q}_{r}(t)$ & $V^{0}(x)$ & $=$ & $\Phi_{l}^{V}(x) q_{l}^{0}$ & & $\boldsymbol{\Phi}_{r}^{V}(x)$ & $=$ & $\Phi_{l}^{V}(x) \mathbf{T}_{l r}$ \\
\hline$\Omega(x, t)$ & $=$ & $\Omega^{0}(x)+\boldsymbol{\Phi}_{r}^{\Omega}(x) \mathbf{q}_{r}(t)$ & $\Omega^{0}(x)$ & $=$ & $\Phi_{l}^{\Omega}(x) q_{l}^{0}$ & , & $\boldsymbol{\Phi}_{r}^{\Omega}(x)$ & $=$ & $\Phi_{l}^{\Omega}(x) \mathbf{T}_{l r}$ \\
\hline$\gamma(x, t)$ & $=$ & $\gamma^{0}(x)+\boldsymbol{\Phi}_{r}^{\gamma}(x) \mathbf{q}_{r}(t)$ & $\gamma^{0}(x)$ & $=$ & $\Phi_{l}^{\gamma}(x) q_{l}^{0}$ & , & $\mathbf{\Phi}_{r}^{\gamma}(x)$ & $=$ & $\Phi_{l}^{\gamma}(x) \mathbf{T}_{l r}$ \\
\hline$\kappa(x, t)$ & $=$ & $\kappa^{0}(x)+\boldsymbol{\Phi}_{r}^{\kappa}(x) \mathbf{q}_{r}(t)$ & $\kappa^{0}(x)$ & $=$ & $\Phi_{l}^{\kappa}(x) q_{l}^{0}$ & , & $\mathbf{\Phi}_{r}^{\kappa}(x)$ & $=$ & $\Phi_{l}^{\kappa}(x) \mathbf{T}_{l r}$ \\
\hline$[3 \times 1]$ & & {$[3 \times 2][2 \times 1]$} & {$[3 \times 1]$} & & {$[3 \times 12][12 \times 1]$} & & {$[3 \times 2]$} & & {$[3 \times 12][12 \times 2]$} \\
\hline
\end{tabular}

Table 5. Assumed modes and time functions for the reduced model

The new variable approximations are listed in Table 5. Substituting the new assumed modes into Eq. (16) yields:

$$
\begin{aligned}
\mathbf{A}_{t r} \dot{\mathbf{q}}_{r}+\mathbf{B}_{t r} \mathbf{q}_{r}+\mathbf{C}_{t r s} \mathbf{q}_{r} \mathbf{q}_{s}+\mathbf{E}_{t u} u_{u}+\mathbf{F}_{t r u} \mathbf{q}_{r} u_{u}+\mathbf{T}_{k t} f_{k}+\mathbf{T}_{k t} m_{k} & =0 \\
y_{y} & =\mathbf{M}_{y r} \mathbf{q}_{r}+y_{y}^{0}
\end{aligned}
$$

where,

$$
\begin{array}{llll}
\mathbf{A}_{t r} & =\mathcal{A}_{k i} \mathbf{T}_{k t} \mathbf{T}_{i r}, & \mathbf{B}_{t r} & =\left[\mathcal{B}_{k i}+\left(\mathcal{C}_{k i j}+\mathcal{C}_{k j i}\right) q_{j}^{0}+\mathcal{F}_{k i u} u_{u}^{0}\right] \mathbf{T}_{k t} \mathbf{T}_{i r} \\
\mathbf{C}_{t r s} & =\mathcal{C}_{k i j} \mathbf{T}_{k t} \mathbf{T}_{i r} \mathbf{T}_{j s}, & \mathbf{E}_{t u} & =\left[\mathcal{E}_{k u}+\mathcal{F}_{k i u} q_{i}^{0}\right] \mathbf{T}_{k t} \\
\mathbf{F}_{t r u} & =\mathcal{F}_{k i u} \mathbf{T}_{k t} \mathbf{T}_{i r}, & \mathbf{M}_{y r} & =\mathcal{M}_{y i} \mathbf{T}_{i r} \\
y_{y}^{0} & =\mathcal{M}_{y i} q_{i}^{0} & &
\end{array}
$$

and, ()$^{0}$ denotes the steady-state solution. Since the modal expansion calculates motion relative to the nonlinear steady state, the steady state solution of the transformed system is always $\mathbf{q}_{r}^{0}=0$. The reduced nonlinear system is the basis for low-order analysis as well as for control design. As $\mathbf{A}_{t r}$ is invertible, the state space formulation of Eq. (21) can be obtained by premultiplying the first equation in Eq. (21) with $\mathbf{A}_{t r}^{-1}$. The state space formulation is:

$$
\begin{aligned}
\dot{\mathbf{q}}_{t} & =\mathrm{B}_{t r} \mathbf{q}_{r}+\mathrm{C}_{t r s} \mathbf{q}_{r} \mathbf{q}_{s}+\mathrm{E}_{t u} u_{u}+\mathrm{F}_{t r u} \mathbf{q}_{r} u_{u}+\mathrm{G}_{k t} f_{k}+\mathrm{H}_{k t} m_{k} \\
y_{y} & =\mathbf{M}_{y r} \mathbf{q}_{r}+y_{y}^{0}
\end{aligned}
$$

where,

$$
\begin{array}{lll}
\mathrm{B}_{t r}=-\mathbf{A}_{t h}^{-1} \mathbf{B}_{h r}, & \mathrm{C}_{t r s}=-\mathbf{A}_{t h}^{-1} \mathbf{C}_{h r s}, & \mathrm{E}_{t u}=-\mathbf{A}_{t h}^{-1} \mathbf{E}_{h u} \\
\mathrm{~F}_{t r u}=-\mathbf{A}_{t h}^{-1} \mathbf{F}_{h r u}, & \mathrm{G}_{k t}=-\mathbf{A}_{k h}^{-1} \mathbf{T}_{h t}, & \mathrm{H}_{k t}=-\mathbf{A}_{k h}^{-1} \mathbf{T}_{h t}
\end{array}
$$

The low order state space model is the basis for the following control design. Even if the model is reduced, from 240 to 12 states, the reduced model still shows good performance. Consider Figure 6 where the natural modes of the full (240 states) and reduced (12 states) model are compared in two plots. The solid lines show the frequencies of the full and the dashed lines the frequencies of the reduced model. For the reduced order model the steady-states are still calculated using the full model but the reduced order models are obtained by using a single set of modeshapes. In the left plot, the frequency variation is calculated under varying rotational speed $\Omega_{3}$ and in the right one under varying external force $f_{3}$ (constant along reference line, rotation speed $\Omega_{3}^{0}=72 \mathrm{rad} / \mathrm{s}$ ). One can see that the natural frequencies are not affected noticeably by 


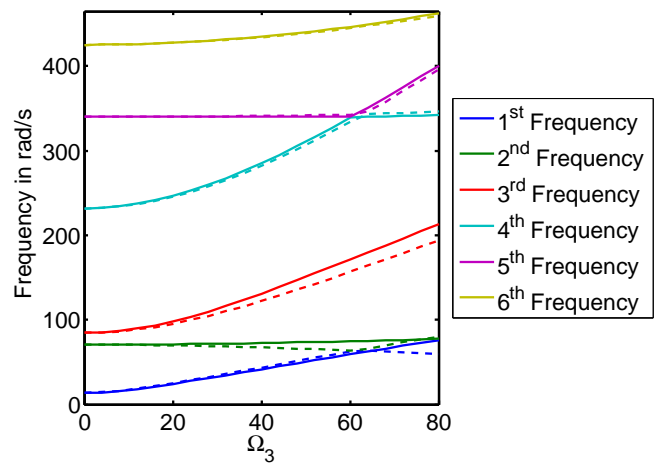

(a) Frequencies under varying rotational speed $\Omega_{3}$

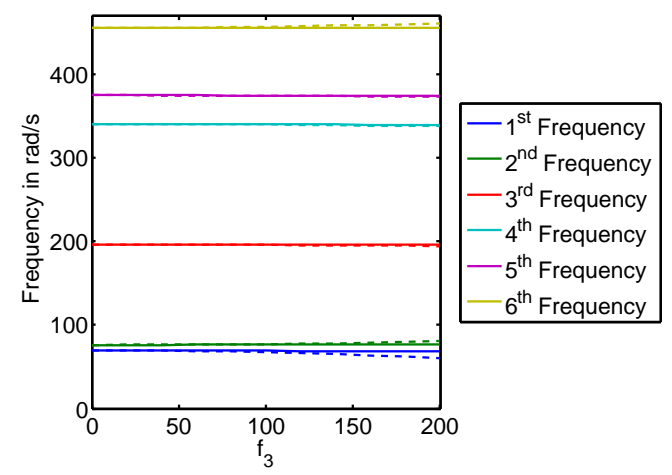

(b) Frequencies under varying external force $f_{3}$

Figure 6. Frequencies of the full and reduced model

a varying external force $f_{3}\left(f_{3}=50 \mathrm{~N} \mathrm{~m}^{-1}\right.$ is the estimated force to lift the helicopter), whereas the rotation speed has a much larger effect. The reduced model is able to track the change in the frequency accurately.

\section{Control Design}

The control design takes advantage of the high fidelity and low order of the reduced blade model Eq. (23). One benefit of the reduced model is, that the state variables can be directly obtained from the measurements, if umodeled frequencies can be perfectly filtered in the measurement signal. The knowledge of the state is utilized to cancel the nonlinearities at a reasonable computational costs. Finally, an energy optimal LQR design of the obtained linear model is performed. The resulting system is guaranteed stable and energy optimal with respect to the resulting linear system.

\section{A. State Extraction}

Based on the good performance of the highly reduced blade model in section $\mathrm{V}$, the number of natural modes for the order reduction can be chosen small. This allows for the calculation of the left pseudo inverse of the measurement matrix $\mathbf{M}_{y r} \in \mathbb{R}^{m \times n}(m>n)$, which is used to obtain the state vector $\mathbf{q}_{r}$ from Eq. (23) as:

$$
\mathbf{q}_{r}=\left(\mathbf{M}_{y r}^{T} \mathbf{M}_{y r}\right)^{-1} \mathbf{M}_{y r}^{T}\left(y_{y}-y_{y}^{0}\right)
$$

It has to be emphasized, that the state extraction shown here based on pseudo inverse, may not work for real world implementations where, the spillover from higher modes will lead to significant error in the static state estimation presented here. Better results can be obtained by a state space observer, when the observer gain is chosen small. Further work is required in the area of state estimation of the nonlinear system.

\section{B. Eliminating Nonlinearities}

The nonlinearities of the blade model are eliminated by defining the new controller output variable $v$. The actuator voltage $u$ is calculated with the new input variable $v$ as:

$$
u_{u}=\left(\mathrm{E}_{t u}+\mathrm{F}_{t r u} \mathbf{q}_{r}\right)^{T}\left[\left(\mathrm{E}_{t u}+\mathrm{F}_{t r u} \mathbf{q}_{r}\right)\left(\mathrm{E}_{t u}+\mathrm{F}_{t r u} \mathbf{q}_{r}\right)^{T}\right]^{-1}\left(-\mathrm{C}_{t r s} \mathbf{q}_{r} \mathbf{q}_{s}+\mathrm{E}_{t u} v_{u}\right)
$$

The cancelling of nonlinearities will also lead to spillover error from higher modes. Inserting Eq. (26) into Eq. (23) yields a linear plant where the measurement equation is omitted due to exact state space extraction.

$$
\dot{\mathbf{q}}_{t}=\mathrm{B}_{t r} \mathbf{q}_{r}+\mathrm{E}_{t u} v_{u}+\mathrm{G}_{k t} f_{k}+\mathrm{H}_{k t} m_{k}
$$


When implementing Eq. (26) in a real environment, one has to compromise between reducing the model to a low order such that the inverse can be calculated efficiently for real-time processing and building a LQR controller for a higher order model without cancelling the nonlinearities. It may be possible to reduce the computational time by approximating the inverse as is done in Quasi-Newton methods for optimization.

\section{LQR Design}

Because of the known state $\mathbf{q}$ of the obtained linear plant (Eq. (27)), an energy optimal least square regulator (LQR) controller ${ }^{17}$ is designed. The cost function determining the LQR of Eq. (27) is:

$$
J=\int_{0}^{\infty}\left[\mathbf{q}^{T} Q \mathbf{q}+v^{T} R v\right] d t \rightarrow \min
$$

where, $Q \geq 0$ and $R>0$ are weighting matrices. $Q$ is weighting the error of the controlled system as $\mathbf{q}=0$ is desired and $R$ is weighting the control effort. Without loss of generality, $R$ is chosen to be identity $(R=I)$ and $Q$ is chosen as:

$$
Q=\frac{1}{2} \alpha \int_{0}^{L} \mathbf{T}^{T}\left[\begin{array}{l}
\Phi^{V} \\
\Phi^{\Omega} \\
\Phi^{\gamma} \\
\Phi^{\kappa}
\end{array}\right]^{T}\left[\begin{array}{cccc}
\mathbb{G} & \mathbb{K} & 0 & 0 \\
\mathbb{K}^{T} & \mathbb{0} & 0 & 0 \\
0 & 0 & \mathbb{U} & \mathbb{V} \\
0 & 0 & \mathbb{V}^{T} & \mathbb{W}
\end{array}\right]\left[\begin{array}{l}
\Phi^{V} \\
\Phi^{\Omega} \\
\Phi^{\gamma} \\
\Phi^{\kappa}
\end{array}\right] \mathbf{T} d x
$$

where, the assumed modes are written in matrix notation. After inserting Eq. (29) into $\mathbf{q}^{T} Q \mathbf{q}$ of Eq. (28) we obtain:

$$
\mathbf{q}^{T} Q \mathbf{q} \stackrel{\text { Table } 2}{\approx} \frac{1}{2} \alpha \int_{0}^{L}\left[\begin{array}{c}
V^{*} \\
\Omega^{*} \\
\gamma^{*} \\
\kappa^{*}
\end{array}\right]^{T}\left[\begin{array}{cccc}
\mathbb{G} & \mathbb{K} & 0 & 0 \\
\mathbb{K}^{T} & \mathbb{0} & 0 & 0 \\
0 & 0 & \mathbb{U} & \mathbb{V} \\
0 & 0 & \mathbb{V} T & \mathbb{W}
\end{array}\right]\left[\begin{array}{c}
V^{*} \\
\Omega^{*} \\
\gamma^{*} \\
\kappa^{*}
\end{array}\right] d x \stackrel{\text { Table } 1}{=} \alpha\left(T^{*}+V^{*}\right)
$$

where, ()$^{*}$ denotes variables measured from the steady state, e.g. $V^{*} \approx \Phi^{V} \mathbf{T} \mathbf{q}$, whereas $V \approx \Phi^{V}\left(\mathbf{T} \mathbf{q}+q^{0}\right)$. Consequently, $T^{*}$ and $U^{*}$ denote a pseudo kinetic and potential energy of the blade. If the steady state solution is zero $\left(q^{0}=0\right), T^{*}=T$ and $U^{*}=U$ are the physical kinetic and potential energy. Equation (28) can be written with Eq. (30) as:

$$
J=\int_{0}^{\infty}\left[\alpha\left(T^{*}+U^{*}\right)+v^{T} v\right] d t \rightarrow \min
$$

The compromise between the blade energy minimization and the minimization of the control effort $v$ can be adjusted by the single parameter $\alpha$. The gain matrix of the resulting state feedback controller is denoted by $K$.

$$
v=-K \mathbf{q}
$$

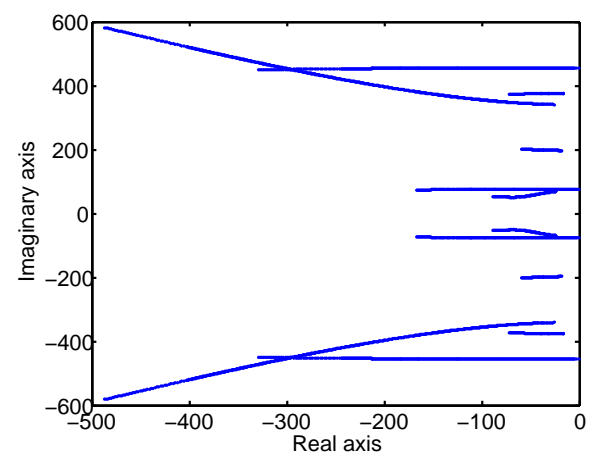

Figure 7. Root locus plot of controlled system poles 


\section{Results}

The control design is tested with a reduced model using the first 6 normal modes which has proved accurate enough in section $\mathrm{V}$. The value for $\alpha$ was chosen to be $10^{8}$ for the simulation. The development of the poles for $\alpha$ values between 1 and $10^{8}$ is shown in Figure 7 .

\begin{tabular}{|ll|cc|cc|}
\hline \hline \multirow{2}{*}{ Mode } & \multicolumn{2}{|c|}{ Controlled Frequencies } & \multicolumn{2}{c|}{ Aeroelastic Frequencies } \\
& & frequency $(\mathrm{rad} / \mathrm{s})$ & damping & frequency $(\mathrm{rad} / \mathrm{s})$ & damping \\
\hline $1^{\text {st }}$ & bending & 53.5352 & $+8.55531 \cdot 10^{-1}$ & 69.4195 & $+3.26373 \cdot 10^{-1}$ \\
$2^{\text {nd }}$ & bending & 200.646 & $+2.82455 \cdot 10^{-1}$ & 196.286 & $+9.35641 \cdot 10^{-2}$ \\
$3^{\text {rd }}$ & bending & 373.476 & $+1.89976 \cdot 10^{-1}$ & 375.224 & $+4.30848 \cdot 10^{-2}$ \\
\hline $1^{\text {st }}$ & lead-lag & 73.1860 & $+9.15802 \cdot 10^{-1}$ & 76.2633 & $+9.82787 \cdot 10^{-4}$ \\
$2^{\text {nd }}$ & lead-lag & 449.795 & $+5.90785 \cdot 10^{-1}$ & 455.697 & $+1.20758 \cdot 10^{-4}$ \\
\hline $1^{\text {st }}$ & torsion & 581.814 & $+6.41581 \cdot 10^{-1}$ & 340.945 & $+7.47685 \cdot 10^{-2}$ \\
\hline \hline
\end{tabular}

Table 6. Natural frequencies of the controlled reduced blade of $12^{\text {th }}$ order

The exact values of the natural frequencies and damping for $\alpha=10^{8}$ are presented in Table 6 . Note, that the controlled system is linear if the nonlinearities are exactly canceled and there is no actuation saturation. It is also pointed out, that the controlled frequencies do not depend only on $\alpha$, but on the order of the reduced model, too. This is because the control strategy may switch if additional modes privilege another strategy.

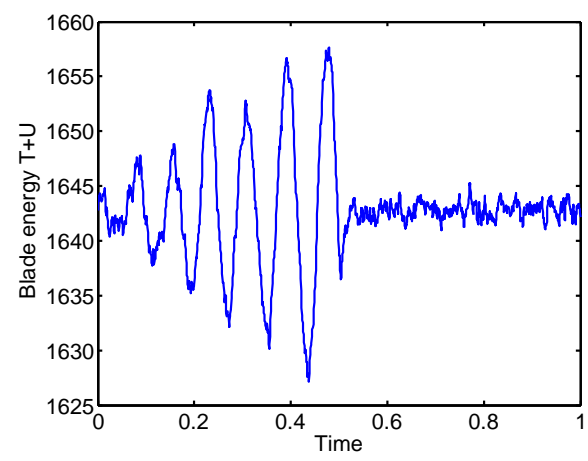

Figure 8. Beam energy $T+U$

In order to validate the controller, a simulation is conducted with the controller switched on after 0.5 seconds. As seen in Figure 8, there is a considerable decrease of the blade energy shortly after the controller is switched on.

Figure 9 shows the effect of the controller on the generalized velocities and strains measured from the steady state. As the generalized strains have their maximum values at the root, they have been measured at the root. The same holds for the generalized velocities that are observed at the tip. The only value that does not improve is $\Omega_{1}$. The controller may use that twist to suppress the velocity $V_{3}$.

Further plots in 10 show the disturbance and the voltages of the actuators. One can see that the voltages do not reach the voltage saturation of $1500 \mathrm{~V}$. The disturbances are modeled as distributed forces only $\left(f^{\text {dist }} \neq 0, m^{\text {dist }}=0\right)$. Furthermore, there is no disturbance $f_{1}^{\text {dist }}$ and $f_{2}^{\text {dist }}$ is less than $f_{3}^{\text {dist }}$. The disturbances are modeled as a sum of Legendre functions that are weighted by white noise signals $f^{d i s t}=$ $P_{l}(\bar{x}) f_{l}^{n o i s e}(t)$. 


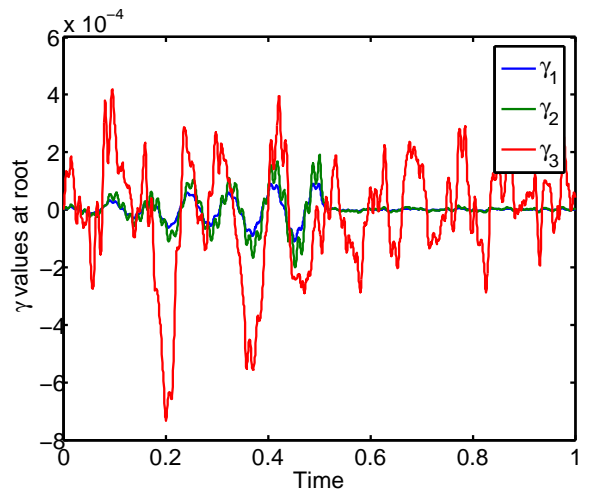

(a) Strain at the root

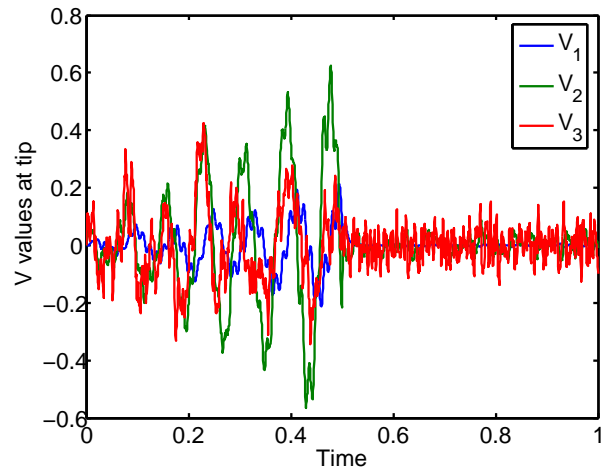

(c) Linear velocity at the tip

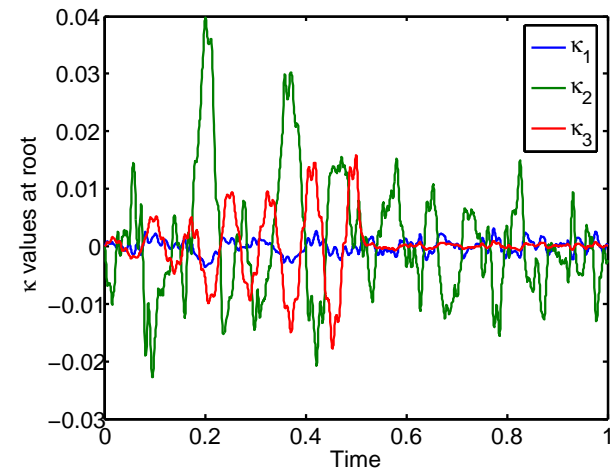

(b) Curvature at the root

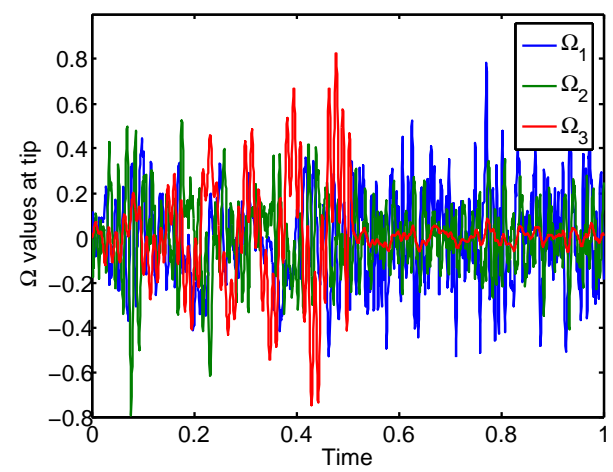

(d) Angular velocity at the tip

Figure 9. Simulation results for $\gamma(\mathbb{D}), \kappa(\mathbb{O}), V(\mathbb{L})$ and $\Omega(\mathbb{L})$

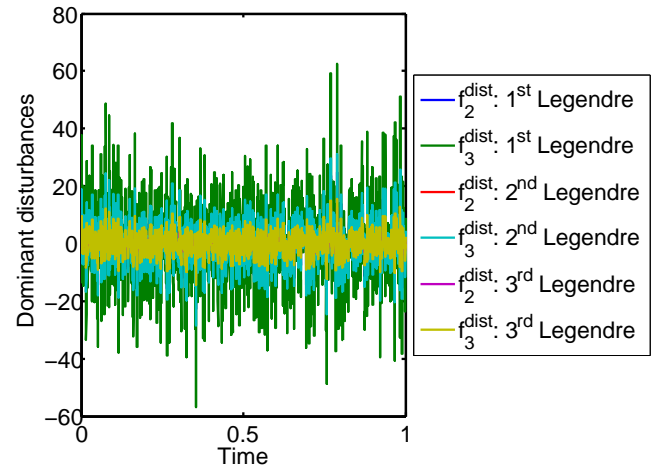

(a) Dominant disturbances

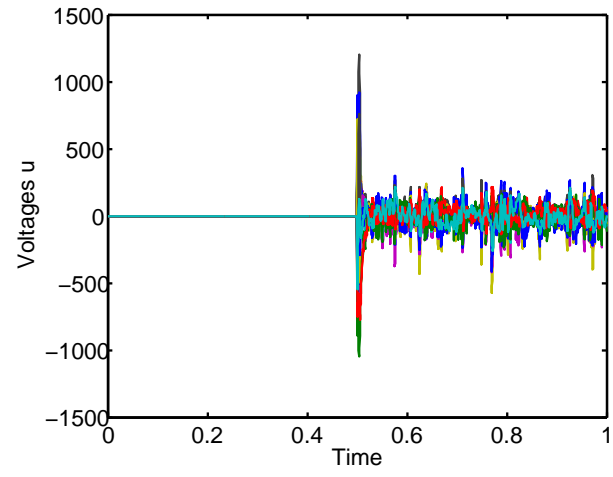

(b) Voltages

Figure 10. Disturbances and Voltages 


\section{Conclusion}

The paper shows an effective way to analyze, simulate and control active helicopter blades. It takes advantage of the good performance of the Galerkin approach which can model the complete nonlinear blade in a quadratic form. The analysis showed the importance of the aerodynamics on the blade model as it adds significant damping. For further analysis (e.g. time marching simulations) a powerful order reduction method has been presented. It exploits the potential of normal modes in capturing the nonlinear blade dynamics. Assuming that the exact known state can be obtained, the possibility of canceling out the blade nonlinearities has been addressed. Finally, an energy optimal LQR design has been performed that provides additional damping for the helicopter blade. Further work is required to address the challenge of estimating the state variables as standard observer design may fail due to unmodeled dynamics.

\section{Appendix}

\section{A. Helicopter Blade Parameters}

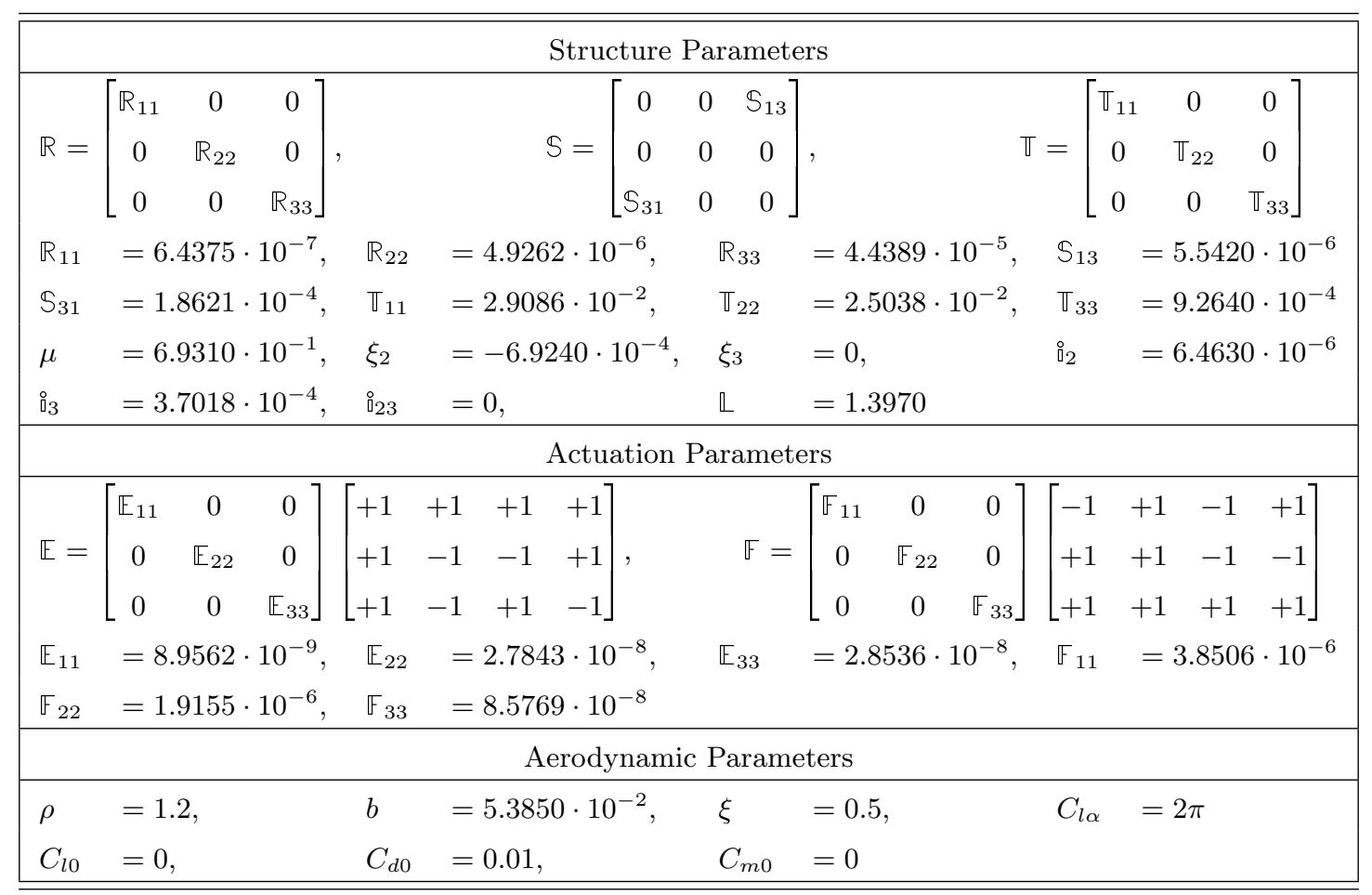

\section{B. Tensors of the Discretized Blade Model}

The tensors of the discretized blade model are given for the special case of a constant cross-section (varying cross-sections are also possible). To ensure a proper formulation of the tensor calculations, a hierarchic tensor notation is introduced.

\section{Hierarchic Tensor Notation}

Consider the approximation $V=\Phi_{i}^{V} q_{i}$ where each $\Phi_{i}^{V}$ is a $3 \times 12$ matrix and each $q_{i}$ is a $12 \times 1$ vector. Now, define $\Phi_{i(a)}$ and $q_{i(a)}$ with $a=1 \ldots 12$ where $V=\Phi_{i(a)}^{V} q_{i(a)}$ is summarized in an inner loop over $a$ and in an outer loop over $i$. Consequently, $\Phi_{i(a)}^{V}$ becomes a $3 \times 1$ vector and $q_{i(a)}$ a scalar. This definition is necessary to e.g. obtain to $\mathcal{C}^{\text {struc }}$-tensor where the tilde operator is used which is defined for $3 \times 1$ vectors only. Note, that for clearness, the hierarchic structure is often suppressed. 


\section{Abbreviations}

In order to obtain a compact formulation of the tensor calculation, some abbreviations are introduced. First, the $\Psi$ constants are introduced:

$$
\Psi^{V}=\left[\begin{array}{llll}
I & 0 & 0 & 0
\end{array}\right], \quad \Psi^{\Omega}=\left[\begin{array}{llll}
0 & I & 0 & 0
\end{array}\right], \quad \Psi^{\gamma}=\left[\begin{array}{llll}
0 & 0 & I & 0
\end{array}\right], \quad \Psi^{\kappa}=\left[\begin{array}{llll}
0 & 0 & 0 & I
\end{array}\right]
$$

where, $I$ is a $3 \times 3$ identity matrix. The abbreviations used for the Legendre functions are:

$$
S_{k}^{\complement}=P_{k}(\mathbb{0}), \quad S_{k}^{\mathbb{L}}=P_{k}(\mathbb{L}), \quad D_{k i}^{\mathbb{D}}=P_{k}(\mathbb{O}) P_{i}(\mathbb{0}), \quad D_{k i}^{\mathbb{\complement}}=P_{k}(\mathbb{L}) P_{i}(\mathbb{L}), \quad S_{k u}^{\mathbb{L} s e g}= \begin{cases}P_{k}(\mathbb{L}), & u=6 \\ 0, & u \neq 6\end{cases}
$$

The abbreviations of the Legendre integrals are:

$$
\begin{array}{rlllll}
S_{k}^{\int} & =\mathbb{L} & \int_{\mathbb{Q}}^{1}\left\{P_{k}(\bar{x})\right\} d \bar{x} & S_{k u}^{\int s e g} & =\mathbb{L} & \int_{(u-1) \frac{1}{6}}^{u \frac{1}{6}}\left\{P_{k}(\bar{x})\right\} d \bar{x} \\
D_{k i}^{\int} & =\mathbb{L} & \int_{\mathbb{O}}^{1}\left\{P_{k}(\bar{x}) P_{i}(\bar{x})\right\} d \bar{x} & { }^{d} S_{k u}^{\int s e g} & = & \int_{(u-1) \frac{1}{6}}^{u \frac{1}{6}}\left\{P_{k}^{\prime}(\bar{x})\right\} d \bar{x} \\
{ }^{d} D_{k i}^{\int} & = & \int_{\mathbb{O}}^{1}\left\{P_{k}(\bar{x}) P_{i}^{\prime}(\bar{x})\right\} d \bar{x} & D_{k i u}^{\int s e g} & =\mathbb{L} & \int_{(u-1) \frac{1}{6}}^{u \frac{1}{6}}\left\{P_{k}(\bar{x}) P_{i}(\bar{x})\right\} d \bar{x} \\
T_{k i j}^{\int} & =\mathbb{L} & \int_{\mathbb{O}}^{1}\left\{P_{k}(\bar{x}) P_{i}(\bar{x}) P_{j}(\bar{x})\right\} d \bar{x} & & &
\end{array}
$$

\section{Tensor Calculations}

$$
\begin{aligned}
& \mathcal{A}_{k(c) i(a)}=D_{k i}^{\mathcal{S}} \quad\left\{\quad \Psi_{(c)}^{V^{T}}\left[\mathbb{G} \Psi_{(a)}^{V}+\mathbb{K} \Psi_{(a)}^{\Omega}\right]+\Psi_{(c)}^{\Omega}{ }^{T}\left[\mathbb{K}^{T} \Psi_{(a)}^{V}+\square \Psi_{(a)}^{\Omega}\right]\right. \\
& \left.+\left(\mathbb{U} \Psi_{(c)}^{\gamma}+\mathbb{V} \Psi_{(c)}^{\kappa}\right)^{T}\left[\Psi_{(a)}^{\gamma}\right]+\left(\mathbb{V}^{T} \Psi_{(c)}^{\gamma}+W_{(c)}^{\kappa}\right)^{T}\left[\Psi_{(a)}^{\kappa}\right]\right\} \\
& \mathcal{B}_{k(c) i(a)}^{n o B C}={ }^{d} D_{k i}^{\mathcal{S}} \quad\left\{\quad \Psi_{(c)}^{V}{ }^{T}\left[-\mathbb{U} \Psi_{(a)}^{\gamma}-\mathbb{V} \Psi_{(a)}^{\kappa}\right]+\Psi_{(c)}^{\Omega}{ }^{T}\left[-\mathbb{V}^{T} \Psi_{(a)}^{\gamma}-\mathbb{W} \Psi_{(a)}^{\kappa}\right]\right. \\
& \left.+\left(\mathbb{U} \Psi_{(c)}^{\gamma}+\mathbb{V} \Psi_{(c)}^{\kappa}\right)^{T}\left[-\Psi_{(a)}^{V}\right]+\left(\mathbb{V}^{T} \Psi_{(c)}^{\gamma}+\mathbb{W} \Psi_{(c)}^{\kappa}\right)^{T}\left[-\Psi_{(a)}^{\Omega}\right]\right\} \\
& +D_{k i}^{\int} \quad\left\{-\Psi_{(c)}^{V^{T}}\left[\tilde{\mathbb{k}}\left(U \Psi_{(a)}^{\gamma}+\mathbb{V} \Psi_{(a)}^{\kappa}\right)\right]-\Psi_{(c)}^{\Omega}{ }^{T}\left[\tilde{\mathbb{k}}\left(\mathbb{V}^{T} \Psi_{(a)}^{\gamma}+\mathbb{W} \Psi_{(a)}^{\kappa}\right)-\tilde{e}_{1}\left(\mathbb{U} \Psi_{(a)}^{\gamma}+\mathbb{V} \Psi_{(a)}^{\kappa}\right)\right]\right. \\
& \left.-\left(\mathbb{U} \Psi_{(c)}^{\gamma}+\mathbb{V} \Psi_{(c)}^{\kappa}\right)^{T}\left[\tilde{k} \Psi_{(a)}^{V}-\tilde{e}_{1} \Psi_{(a)}^{\Omega}\right]-\left(\mathbb{V}^{T} \Psi_{(c)}^{\gamma}+\mathbb{W} \Psi_{(c)}^{\kappa}\right)^{T}\left[\tilde{k} \Psi_{(a)}^{\Omega}\right]\right\} \\
& \mathcal{C}_{k(c) i(a) j(b)}^{s t r u c}=T_{k i j}^{\int} \quad\left\{\quad \Psi_{(c)}^{V}{ }^{T}\left[-\tilde{\Psi}_{(a)}^{\kappa}\left(\mathbb{U} \Psi_{(b)}^{\gamma}+\mathbb{V} \Psi_{(b)}^{\kappa}\right)+\tilde{\Psi}_{(a)}^{\Omega}\left(\mathbb{G} \Psi_{(b)}^{V}+\mathbb{K} \Psi_{(b)}^{\Omega}\right)\right]\right. \\
& +\Psi_{(c)}^{\Omega T}\left[\tilde{\Psi}_{(a)}^{\Omega}\left(\mathbb{K}^{T} \Psi_{(b)}^{V}+\square \Psi_{(b)}^{\Omega}\right)+\tilde{\Psi}_{(a)}^{V}\left(\mathbb{G} \Psi_{(b)}^{V}+\mathbb{K} \Psi_{(b)}^{\Omega}\right)-\tilde{\Psi}_{(a)}^{\kappa}\left(\mathbb{V}^{T} \Psi_{(b)}^{\gamma}\right.\right. \\
& \left.\left.+\mathbb{W} \Psi_{(b)}^{\kappa}\right)-\tilde{\Psi}_{(a)}^{\gamma}\left(U \Psi_{(b)}^{\gamma}+\mathbb{V} \Psi_{(b)}^{\kappa}\right)\right] \\
& \left.+\left(\mathbb{U} \Psi_{(c)}^{\gamma}+\mathbb{V} \Psi_{(c)}^{\kappa}\right)^{T}\left[-\tilde{\Psi}_{(a)}^{\kappa} \Psi_{(b)}^{V}-\tilde{\Psi}_{(a)}^{\gamma} \Psi_{(b)}^{\Omega}\right]+\left(\mathbb{V}^{T} \Psi_{(c)}^{\gamma}+\mathbb{W} \Psi_{(c)}^{\kappa}\right)^{T}\left[-\tilde{\Psi}_{(a)}^{\kappa} \Psi_{(b)}^{\Omega}\right]\right\}
\end{aligned}
$$

The tensor calculating the aerodynamic force and moment is:

$$
\begin{aligned}
& \mathcal{C}_{k(c) i(a) j(b)}^{a e r o}=T_{k i j}^{S}\left\{\quad \Psi_{(c)}^{V}\left\{\begin{array}{lll}
0 & 1 & 0
\end{array}\right\}^{T}\left[\Psi_{(a)}^{V}{ }^{T} \mathbb{X}^{V V} \Psi_{(b)}^{V}+\Psi_{(a)}^{V}{ }^{T} \mathbb{X}^{V \Omega} \Psi_{(b)}^{\Omega}+\Psi_{(a)}^{\Omega}{ }^{T} \mathbb{X}^{\Omega \Omega} \Psi_{(b)}^{\Omega}\right]\right. \\
& +\Psi_{(c)}^{V}\left\{\begin{array}{lll}
{ }^{T} & 0 & 1
\end{array}\right\}^{T}\left[\Psi_{(a)}^{V}{ }^{T} \mathbb{\mho}^{V V} \Psi_{(b)}^{V}+\Psi_{(a)}^{V}{ }^{T} \mathbb{Y}^{V}{ }^{V} \Psi_{(b)}^{\Omega}\right] \\
& \left.+\Psi_{(c)}^{\Omega}\left\{\begin{array}{lll}
1 & 0 & 0
\end{array}\right\}^{T}\left[\Psi_{(a)}^{V}{ }^{T} \mathbb{Z}^{V V} \Psi_{(b)}^{V}+\Psi_{(a)}^{V}{ }^{T} \mathbb{Z}^{V \Omega} \Psi_{(b)}^{\Omega}\right]\right\}
\end{aligned}
$$


The actuation for constant cross-section is determined by:

$$
\begin{aligned}
& \mathcal{E}_{k(c) u}^{n o B C}={ }^{d} S_{k u}^{\int s e g} \quad\left\{\quad \Psi_{(c)}^{V}{ }^{T}(\mathbb{U E}+\mathbb{V} \mathbb{F})+\Psi_{(c)}^{\Omega T}\left(\mathbb{V}^{T} \mathbb{E}+\mathbb{W} \mathbb{F}\right)\right\} \\
& +S_{k u}^{\int \operatorname{seg}}\left\{\quad \Psi_{(c)}^{V}{ }^{T}[\tilde{\mathbb{k}}](\mathbb{U E}+\mathbb{V} \mathbb{E})+\Psi_{(c)}^{\Omega}[\tilde{\mathbb{k}}]\left(\mathbb{V}^{T} \mathbb{E}+\mathbb{W} \mathbb{F}\right)+\Psi_{(c)}^{\Omega}\left[\tilde{e}_{1}\right](\mathbb{U} \mathbb{E}+\mathbb{V} \mathbb{F})\right\} \\
& \mathcal{F}_{k(c) i(a) u}^{n o B C}=D_{k i u}^{\int s e g} \quad\left\{\quad \Psi_{(c)}^{V}{ }^{T}\left[\tilde{\Psi}_{(a)}^{\kappa}\right](\mathbb{U} \mathbb{E}+\mathbb{V} \mathbb{F})+\Psi_{(c)}^{\Omega T}\left[\tilde{\Psi}_{(a)}^{\kappa}\right]\left(\mathbb{V}^{T} \mathbb{E}+\mathbb{W} \mathbb{F}\right)+\Psi_{(c)}^{\Omega}{ }^{T}\left[\tilde{\Psi}_{(a)}^{\gamma}\right](\mathbb{U} \mathbb{E}+\mathbb{V} \mathbb{F})\right\}
\end{aligned}
$$

$f_{k}$ and $m_{k}$ are:

$$
\begin{aligned}
& f_{k(c)}=S_{k}^{\int}\left\{-\Psi_{(c)}^{V^{T}} f\right\} \\
& m_{k(c)}=S_{k}^{\int}\left\{-\Psi_{(c)}^{\Omega T} m\right\}
\end{aligned}
$$

The tensors occurring due to boundary conditions are:

$$
\begin{aligned}
& \begin{aligned}
\mathcal{D}_{k(c)}^{B C}= & S_{k}^{\mathbb{Q}} \\
+ & +S_{k}^{\mathbb{Q}}
\end{aligned}\left\{\begin{aligned}
& \left(\mathbb{U} \Psi_{(c)}^{\gamma}+\mathbb{V} \Psi_{(c)}^{\kappa}\right)^{T} V^{\mathbb{Q}}+\left(\mathbb{V}^{T} \Psi_{(c)}^{\gamma}+\mathbb{W} \Psi_{(c)}^{\kappa}\right)^{T} \Omega^{\mathbb{D}}
\end{aligned}\right\} \\
& \begin{aligned}
\mathcal{B}_{k(c) i(a)}^{B C}= & D_{k i}^{\mathbb{Q}} \\
& +D_{k i}^{\mathbb{\complement}}
\end{aligned}\left\{\begin{aligned}
- & \left.\left(\mathbb{U} \Psi_{(c)}^{\gamma}+\mathbb{V} \Psi_{(c)}^{\kappa}\right)^{T}\left[\Psi_{(a)}^{V}\right]-\left(\mathbb{V}^{T} \Psi_{(c)}^{\gamma}+\mathbb{W} \Psi_{(c)}^{\kappa}\right)^{T}\left[\Psi_{(a)}^{\Omega}\right]\right\} \\
& \left.\Psi_{(c)}^{V}{ }^{T}\left[\mathbb{U} \Psi_{(a)}^{\gamma}+\mathbb{V} \Psi_{(a)}^{\kappa}\right]+\Psi_{(c)}^{\Omega}{ }^{T}\left[\mathbb{V}^{T} \Psi_{(a)}^{\gamma}+\mathbb{W} \Psi_{(a)}^{\kappa}\right]\right\}
\end{aligned}\right. \\
& \mathcal{E}_{k(c) u}^{B C}=S_{k u}^{\mathbb{L} \text { seg }} \quad\left\{-\Psi_{(c)}^{V}{ }^{T}(\mathbb{U} \mathbb{E}+\mathbb{V} \mathbb{F})-\Psi_{(c)}^{\Omega T}\left(\mathbb{V}^{T} \mathbb{E}+\mathbb{W} \mathbb{F}\right)\right\}
\end{aligned}
$$

The measurement tensors is:

$$
\mathcal{M}_{y i(a)}=\left\{\begin{array}{l}
\Psi^{\gamma} \\
\Psi^{\kappa}
\end{array}\right\} P_{i(a)}\left(\frac{y-1}{4}\right)
$$

\section{References}

${ }^{1}$ Shin, S. J. and Cesnik, C. E. S., "Helicopter Performance and Vibration Enhancement by TwistActuated Blades," AIAA, April 2003.

${ }^{2}$ Wilbur, M. L., Mirick, P. H., Yeager, W. T., Langston, C. W., Cesnik, C. E. S., and Shin, S., "Vibratory loads reduction testing of the NASA/Army/MIT active twist rotor," Journal of the American Helicopter Society, Vol. 47, No. 2, Apr. 2002, pp. $123-133$.

${ }^{3}$ Traugott, J. P., Patil, M. J., and Holzapfel, F., "Nonlinear Dynamics and Control of Integrally Actuated Helicopter Blades," Proceedings of the 13th Adaptive Structures Conference, Austin, Texas, April 2005, AIAA-2005-2271.

${ }^{4}$ Patil, M. J. and Althoff, M., "Energy-consistent, Galerkin approach for the nonlinear dynamics of beams using mixed, intrinsic equations," Proceedings of the AIAA/ASME/ASCE/AHS/ASC Structures, Structural Dynamics and Materials Conference, Newport, Rhode Island, May 2006.

${ }^{5}$ Patil, M. J. and Hodges, D. H., "Flight Dynamics of Highly Flexible Flying Wings," Proceedings of the International Forum on Aeroelasticity and Structural Dynamics, Munich, Germany, June 2005.

${ }^{6}$ Hodges, D. H., "A Mixed Variational Formulation Based on Exact Intrinsic Equations for Dynamics of Moving Beams," International Journal of Solids and Structures, Vol. 26, No. 11, 1990, pp. 1253 - 1273.

${ }^{7}$ Hodges, D. H., "Geometrically Exact, Intrinsic Theory for Dynamics of Curved and Twisted Anisotropic Beams," AIAA Journal, Vol. 41, No. 6, 2003, pp. 1131-1137.

${ }^{8}$ Hopkins, A. S. and Ormiston, R. A., "An Examination of Selected Problems in Rotor Blade Structural Mechanics and

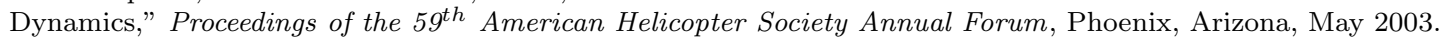

${ }^{9}$ Patil, M. J. and Johnson, E. R., "Cross-sectional Analysis of Anisotropic, Thin-Walled, Closed-Section Beams with Embedded Strain Actuation," Proceedings of the 13th Adaptive Structures Conference, Austin, Texas, April 2005, AIAA-20052037.

${ }^{10}$ Cesnik, C. E. S. and Palacios, R., "Modeling Piezocomposite Actuators Embedded in Slender Structures," Proceedings of the 44th Structures, Structural Dynamics, and Materials Conference, Norfolk, Virginia, April 2003, AIAA Paper $2003-1803$.

${ }^{11}$ Peters, D. A. and Johnson, M. J., "Finite-State Airloads for Deformable Airfoils on Fixed and Rotating Wings," Symposium on Aeroelasticity and Fluid/Structure Interaction, Proceedings of the Winter Annual Meeting, ASME, November 6 - 11, 1994. 
${ }^{12}$ Fletcher, C. A. J., Computational Galerkin Methods, Springer, 1984.

${ }^{13}$ Abramowitz, M. and Stegun, A., Handbook of Mathematical Functions, Dover, 1964.

${ }^{14}$ Patil, M. J., "Decoupled Second-Order Equations and Modal Analysis of a General Non-Conservative System," Proceedings of the AIAA Dynamics Specialists Conference, Atlanta, Georgia, April 2000, AIAA-2000-1654.

${ }^{15}$ Noor, A. K. and Peters, J. M., "Reduced Basis Technique for Nonlinear Analysis of Structures," AIAA Journal, Vol. 19, No. 4, Apr. 1980.

${ }^{16}$ Bauchau, O. A. and Guernsey, D., "On the Choice of Appropriate Bases for Nonlinear Dynamic Modal Analysis," Journal of the American Helicopter Society, Vol. 38, No. 4, Oct. 1993, pp. $28-36$.

${ }^{17}$ Burl, J. B., Linear Optimal Control, Prentice Hall, November 1998. 